\title{
A predictor for predicting Escherichia coli transcriptome and the effects of gene perturbations
}

\author{
Maurice HT Ling ${ }^{*}$ and Chueh Loo Poh ${ }^{*}$
}

\begin{abstract}
Background: A means to predict the effects of gene over-expression, knockouts, and environmental stimuli in silico is useful for system biologists to develop and test hypotheses. Several studies had predicted the expression of all Escherichia coli genes from sequences and reported a correlation of 0.301 between predicted and actual expression. However, these do not allow biologists to study the effects of gene perturbations on the native transcriptome.

Results: We developed a predictor to predict transcriptome-scale gene expression from a small number $(n=59)$ of known gene expressions using gene co-expression network, which can be used to predict the effects of over-expressions and knockdowns on E. coli transcriptome. In terms of transcriptome prediction, our results show that the correlation between predicted and actual expression value is 0.467 , which is similar to the microarray intra-array variation ( $p$-value $=0.348$ ), suggesting that intra-array variation accounts for a substantial portion of the transcriptome prediction error. In terms of predicting the effects of gene perturbation(s), our results suggest that the expression of $83 \%$ of the genes affected by perturbation can be predicted within $40 \%$ of error and the correlation between predicted and actual expression values among the affected genes to be 0.698 . With the ability to predict the effects of gene perturbations, we demonstrated that our predictor has the potential to estimate the effects of varying gene expression level on the native transcriptome.
\end{abstract}

Conclusion: We present a potential means to predict an entire transcriptome and a tool to estimate the effects of gene perturbations for E. coli, which will aid biologists in hypothesis development. This study forms the baseline for future work in using gene co-expression network for gene expression prediction.

\section{Background}

One of the key challenges in systems biology is to develop a complete computational model of biology that can be used for both integration of knowledge and to develop and test hypotheses. A number of computational tools had been developed (reviewed in [1]) over the years, such as COBRA toolkit [2]. However, Medema et al. [1] did not mention about any tools for transcriptome prediction. Selinger et al. [3] proposed that a means to predict gene expressions will be useful for predicting the effects of gene over-expression, knockouts, and environmental stimuli.

A number of recent studies had attempted to predict gene expression using in silico methods. Chikina et al. [4] used microarray data to predict tissue-specific gene

\footnotetext{
* Correspondence: mauriceling@acm.org; clpoh@ntu.edu.sg

School of Chemical and Biomedical Engineering, Nanyang Technological University, Nanyang Ave, Singapore, Singapore
}

expression in various tissues of Caenorhabditis elegans. Ouyang et al. [5] used transcription factors binding data from ChIP-seq experiments to predict gene expression in mouse embryonic cells. McLeay et al. [6] expanded on Ouyang et al. [5] by modeling the binding efficiency of transcription factors to promoters; thereby, using it to predict gene expressions. McLeay et al. [6] reported correlation of 0.64 when tested on GM12878 cells but histones modification and chromatin accessibility data needs to be incorporated, which may limit its application due to the lack of required data. Fox and Erill [7] used relative codon usage bias to predict the expression levels of $E$. coli genes of more than $1000 \mathrm{bp}$, achieving a correlation of 0.489 between predicted and actual expression. This is higher than the correlation of 0.301 reported by Roymondal et al. [8] when correlating relative codon usage bias to the expression levels of $E$. coli genes of all lengths. A 
further study by the same group attempted to predict the expression of Synechocystis PCC 6803 (a cyanobacterium) using relative codon usage bias reported a correlation between 0.240 and 0.356 [9]. However, there had been no study demonstrating the use of gene co-expression network (GCN) in gene expression prediction in E. coli.

GCN had been commonly used to study expressional similarities of genes [10], where the nodes are the genes and a link (an edge) between 2 nodes when the gene-pair is co-expressed. The basis of $\mathrm{GCN}$ is that expressionally correlated genes are likely to be functionally related $[11,12]$ or evolutionarily conserved $[13,14]$. GCN had been successfully used in several cases, such as identifying developmental processes [15], annotating functional genes [16], and studying disease progression [17]. Although there had been a number of methods proposed to estimate the degree of co-expression [18]; such as using rank correlation [19], weights [20] and mixed-models [21]; Pearson's correlation is commonly used $[4,11,22,23]$ due to presence of upper and lower boundaries of correlation coefficient, resulting in ease of interpretation [24]. Once the co-expression between two genes is established, the expression level of a gene can be predicted from the known expression of another gene by means of linear regression [25]. This suggests that GCN has the advantage of estimating a large number of gene expressions from a small number of known gene expressions.

In this study, we developed a predictor to predict transcriptome-scale gene expression from a small number of known gene expressions using GCN, which may be used to predict the effects of over-expressions and knockdowns on E. coli transcriptome. The correlation of 21 genes that are detected by 2 probes on the microarray is 0.490 . Using microarray data not used in GCN building, our transcriptome prediction results show that the correlation between expected and predicted expressions using expression values is 0.467 . Our perturbation prediction results show that the correlation between predicted and actual expression values among perturbation-affected genes to be 0.698 . Using our ability to predict the effects of gene perturbations, we presented a case study to estimate the effects of varying gene expression level of hydrogenase 2 maturation endopeptidase $(h y b D)$; thereby, identifying a range of expression levels in which there is no effect on the native transcriptome and we termed this range as expressional buffer. Hence, this study presents a potential means to estimate transcriptome-scale gene expressions which has the potential to predict the effects of gene over-expression, knockouts, and environmental stimuli [3].

\section{Results and discussion}

We developed a predictor based on GCN to predict transcriptome-scale gene expression and estimate the effects of changing the expression of genes, such as overexpression and under-expression, on a native transcriptome.

\section{Fifty-nine source genes reach 6140 genes}

A total of 51,121,216 permuted probe-pairs were generated from 10,112 non-control probes in GPL3154. These non-control probes were mapped to 10,091 genes. Thus, only 21 genes were represented by 2 probes (given in Additional file 1: Table S2). For simplicity, we shall use "genes" to represent both "genes" and "probes" hereafter. The average correlation of these gene-pairs is 0.027 , which is similar to that reported in other studies [14,26]. Using the correlation threshold suggested by Reverter et al. [27] of absolute correlation coefficient that is higher than 0.75 (p-value $=1.28 \mathrm{e}-102$ after Bonferroni correction), only $533,311(1.04 \%)$ pairs and 7,360 (72.78\%) genes remained and were used to construct the co-expression network.

Using the 21 genes that were represented by 2 probes on the microarray, intra-array variation [28] can be estimated by analyzing the differences from these 2 probes [29]. Theoretically, their expression values will be the same and the ratio of expression values will be 1 , which can be translated to perfect correlation, as they are measuring the same transcript. Using all 605 microarrays, our results suggest that the average correlation is 0.490 with a standard error of 0.0488 . This is similar to the correlation of 0.535 ( $\mathrm{p}$-value $=0.36$, power $>0.99$ ) reported by Ling et al. [14] on microarray technical replicates of identical biological samples. The average deviation [ $\left(\sum_{i=1}^{N}\right.$ |average ratio- $\left.1 \mid\right) / N$ ] from a perfect ratio of 1 is $19.19 \%$, suggesting that the average intra-array variation can be estimated to be $19.19 \%$ (Additional file 1: Table S2). Our estimate falls within 11\% and 33\% intraarray variation estimated by Anderson et al. [30] whom proposed a novel Array Microenvironment Normalization $(\mathrm{AMN})$ to reduce $72 \%$ of the intra-array variation. However, Gyorffy et al. [31] demonstrated that results from RMA (Robust Multi-array Average) normalization correlates well with both tissue samples and cell lines even though other normalization schemes appears to work better with tissue samples or cell lines independently. In addition, AMN has not been shown to correlate well with quantitative PCR results. Hence, considering that RMA normalization correlates well with both tissue samples and cell lines, we chose to continue with RMA normalized data. Nevertheless, our estimated intra-array variation of $19.19 \%$ suggests potential area of future studies in normalization techniques aiming at reducing such variation as intra-array variation represents noise in the source data which may affect downstream analyses [32].

After GCN construction, the next step was to determine a small set of genes with the maximum network coverage 
and minimum degree of separation (also known as jump) as network coverage is directly proportional to the extent of predictable transcriptome and the error in prediction is directly proportional to the number of jumps. We analyzed the number of jumps between any given genepairs. Our results suggest that the density peaks at 4 jumps (Additional file 1: Figure S1). With reference to Figure 1, when a pair of genes is linked by a finite number of paths, the expression of one of the pairs (known as a target gene) can be predicted if expression of the other gene (known as a source gene) is known. As there can be many paths between the source and target genes, there can be many predicted expression values for the target gene as the number of predicted values equals the number of paths. Our results show that accuracy at $20 \%$ error decreases drastically from path length of 5 or more (Figure 2A), suggesting that the limits of predictability is 4 jumps. Although it can be argued that path lengths of 2 or 3 may yield higher accuracy, the number of source genes will increase as the number of source genes needed is inversely proportional to the path length in order to achieve the same network coverage. In addition, our results also suggest that intra-array variation adversely affect prediction accuracy (Figure 2B).

We analyzed the degree of network coverage using 2 sets of source probes - a set of 32 source genes from coefficient of determination $\left(\mathrm{r}^{2}\right)$ of more than 0.95 and a set of 392 source genes from absolute Pearson's correlation of more than 0.95 . Since coefficient of variation can be used as a measure of prediction accuracy between a pair of source and target probes, strong correlation in the first jump is likely to improve the overall prediction. Our results show that the coverage from the set of 392 genes is significantly better than that of 32 genes (Figure 3). However, 392 is a large number of genes to measure experimentally. We analyzed this set of 392 genes in order to reduce it into a smaller set of marker genes [33] which is feasible for experimental work. At 4 jumps, a number of these genes reach to the same set of target genes. By removing redundancy, we reduced 392 genes to 49 genes but the coverage dropped from 6154 to 6053 genes. We examined the set of genes not reached by these 49 source genes within 4 jumps and added 10 genes with the highest degree (most number of edges) to increase the number of source genes from 49 to 59 . With this addition, the coverage increases to 6140 genes. We argue that adding more source probes at this stage is unlikely to give equivalent increase in coverage. Hence, we proceeded with a set of 59 source genes (see Additional file 1: Table S3 for the description of these 59 genes).

\section{Transcriptome predicted within $40 \%$ error using 59 source genes}

We attempted to predict $E$. coli transcriptome using the panel of 59 source genes. To do so, we implemented a single pass transcriptome predictor where each target gene will be predicted using expression value from one source gene. We evaluated the accuracy of our predictor using a set of 30 microarrays from experiments not used in the GCN construction (see Additional file 1: Table S4 for the microarrays used). These sets of microarray data had been published in 25 different experimental studies [34-58]; thus, representing a set of unbiased data for evaluating the performance of our predictor (see Additional file 1: Table S4 for details of experiments). In this aspect, we hold the same evaluation principles as Abadia et al. [59], whom used data from various centres worldwide to evaluate the performance of a newly developed protocol.

Although these 30 microarrays originated from a diverse range of studies, several recent studies [60-62] had

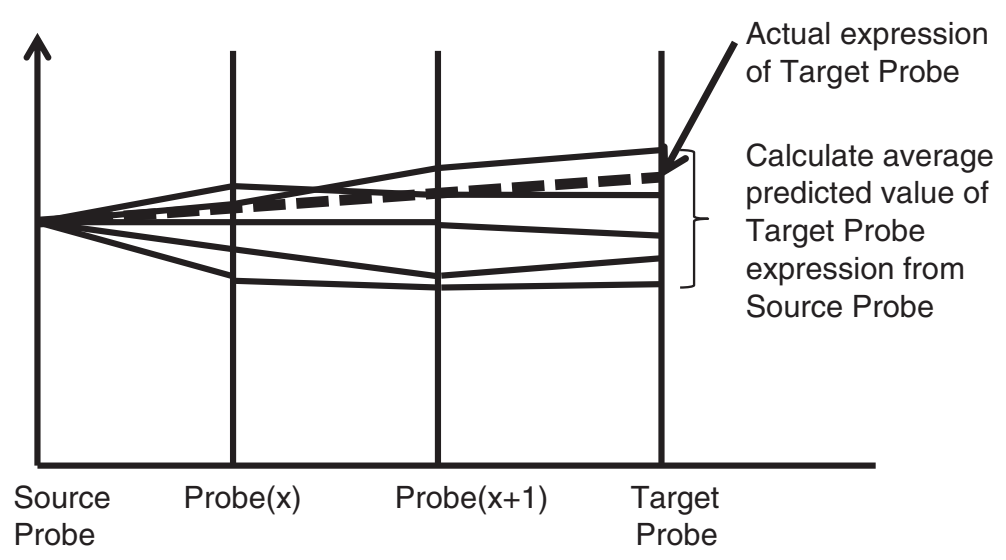

Figure 1 Concept in predicting target gene expression value. Target gene expression value can be estimated from a single source gene expression based on linear regression. In this figure, the source probe/gene and target probe/gene are separated by 2 sets of probe/genes and 5 different paths. Expression values of probes/genes adjacent to the source probe/gene [denoted as Probe(x)] can be estimated by linear regression. This can be done repeatedly to reach the target. Target probe/gene expression value can then be statistically inferred. 
(A) Path Length against Accuracy

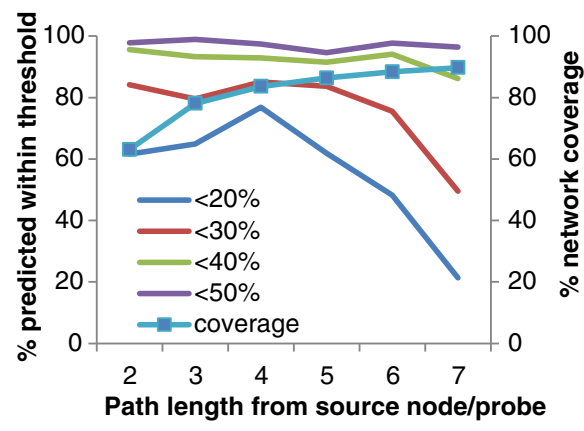

(B) Effects of Intra-Array Variation

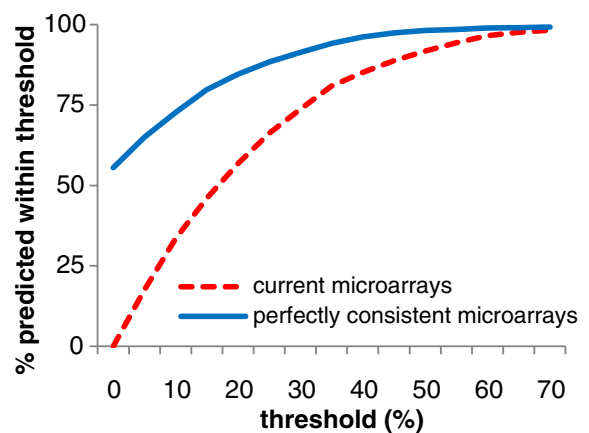

Figure 2 Precision of prediction. Panel A shows the percentage of genes predicted within specific thresholds from 2 to 7 jumps from the source gene. The predicted value of each gene is defined as the average predicted values from source gene to target gene across different paths. If the actual expression value of a gene is $+/-20 \%$ the predicted value, then the gene is considered to be predicted within $20 \%$ threshold. The network coverage from 392 source genes (see Figure 3) is shown. Panel $\mathbf{B}$ shows the difference in prediction precision between the current set of microarray with 19.19\% intra-slide variation and a set of theoretically consistent microarray with no intra-slide variation.

suggested that published microarray datasets contain value beyond their initial studies. For example, several studies had analyzed published microarray datasets for reference genes $[63,64]$ and other biologically significant features [65]. Moreover, most of the 30 microarrays originated from studies that were representative of the type of experimental studies which we expect our predictor to be useful in. For example, Traxler et al. [56] examined the global effects of amino acid starvation in E. coli MG1655 and Lee et al. [48] examined the expression of $E$. coli stress-related proteins in the presence of pollutants. Hence, our evaluation also represented 30 experimental case studies on the use of our transcriptome predictor.
Our results suggest a positive correlation between the average predicted expression values and the actual expression values of each target gene across all 30 transcriptomes (average correlation $=0.467$, standard error $(\mathrm{SE})=0.0383$, $\mathrm{p}$-value $=2.77 \mathrm{e}-13)$. This is similar to the correlation of 0.489 ( $\mathrm{p}$-value $=0.656$ ) reported by Fox and Erill [7] using relative codon usage bias to predict the expression levels of $E$. coli genes of more than $1000 \mathrm{bp}$ and higher than the reported correlation of 0.240 to 0.356 ( $\mathrm{p}$-value $<0.031$ ) in a study using codon usage bias to predict expression of Synechocystis PCC 6803 genes [9]. As our predictor is not restricted to the length of gene that can be predicted as in the case of Fox and Erill [7], the correlation of 0.301 between actual

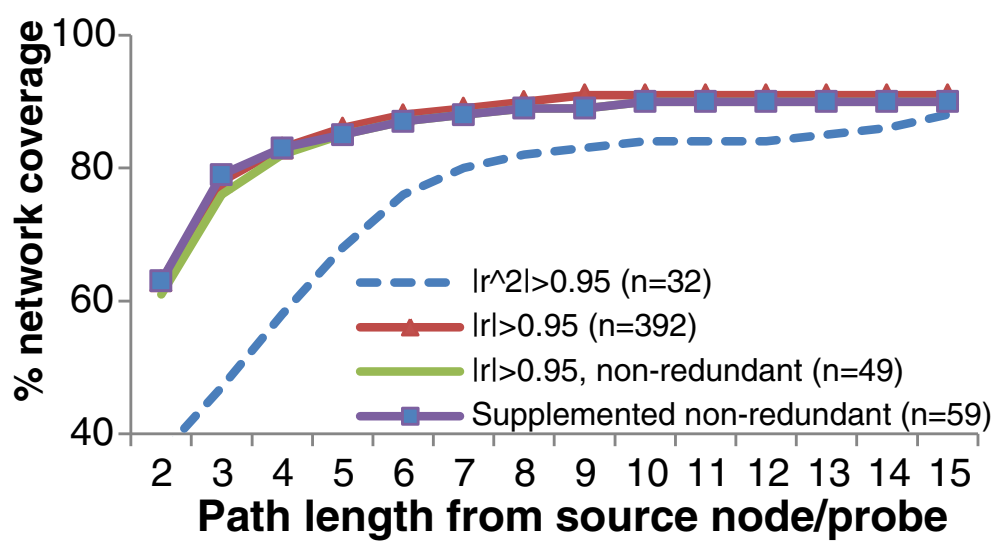

Figure 3 Network coverage by path length. The number of target genes reachable by 32 source genes (from a threshold of $\left.\left|r^{2}\right|>0.95\right)$ is much lesser than that reachable by 392 source genes (from a threshold of $|r|>0.95$ ) at a path length of 4 . However, using 392 source genes is not practical for experimental validation. By removing redundant source genes, we arrived at 49 source genes but at a loss of 101 target genes. By supplementing with 10 genes with high degrees (total of 59 source genes), we increased the reach by 87 target genes, giving a total of 6140 reachable target genes from 59 source genes. 
and predicted expression reported by Roymondal et al. [8] is a more accurate comparison to our result as Roymondal et al. [8] use relative codon usage bias to predict the expression levels of all $E$. coli genes instead of those more than $1000 \mathrm{bp}$. Based on this, our predictor is more accurate $(p$-value $=0.0002)$ than that reported by Roymondal et al. [8]. In addition, this is not significantly different from the correlation of 0.490 between duplicate probes from the microarray data measuring the same transcript ( $p$-value $=0.613$ ), suggesting that intra-array variation accounts for a substantial portion of transcriptome prediction error.

Our results show that 24 of the 30 transcriptomes (see Additional file 1: Table S4 for the microarrays used) are predicted within $40 \%$ error using 30 to 10000 paths between each source and target gene (Figure 4), with the average error of $34.29 \%$ (standard error $=2.807 \%$ ). This is comparable to $33 \%$ error ( $\mathrm{p}$-value $=0.65$ ) using chromatin states and transcription factor occupancy, which are less readily available than gene expression values, to predict spatial-temporal expression of genes [66]. Hence, our predictor can potentially be used as a preliminary in silico hypothesis screening tool, which only requires the expression of a panel of source genes and can be obtained with routine experimental tools such as quantitative PCR, prior to full-scale transcriptome analysis (Figure 5).

Many studies use experimental techniques, such as PCRbased techniques, on a small set of genes to validate microarray results. Kendall et al. [45] use quantitative PCR for detailed analysis of microarray findings elucidated by comparing the transcriptomes of wild-type $E$. coli 86-24 strain and luxS mutant VS94, which corresponds to GSM180104 and GSM180102 respectively. These 2 microarrays have not been used in our GCN construction. Hence, we predicted the transcriptomes of E. coli 86-24 strain and luxS mutant VS94 using source gene expressions from GSM180104 and GSM180102 respectively. We compare our prediction results with 10 quantitative PCR results of Kendall et al. [45], showing 3 of the 10 evaluated genes to be differentially expressed. Our results suggest 8 out of 10 matched conclusions with one false positive and false negative each (Additional file 1: Table S5).

Although our results show that the predicted gene expressions of 30 representative test samples are more accurate than that of Roymondal et al. [8], our results also show that only 24 of the 30 transcriptomes can be predicted within $40 \%$ error and 8 out of 10 findings using our prediction match quantitative PCR results of Kendall et al. [45]. Despite using 30 representative test samples for our evaluation, our results are based on meta-analysis of published data. Using meta-analysis of published experimental data, we have shown the potential of the predictor. However, the protocol will need to be further validated using more condition-specific experiments. At the moment, our study forms a baseline towards this direction.

It is conceivable that using more than one source gene to predict a target gene may improve prediction accuracy. To test this hypothesis, we developed a multi-pass transcriptome predictor that allows for the use of any number of source genes to predict a target gene. Network coverage analysis shows that 59 source genes can reach a total of 169,012 genes in 4 jumps or each target gene is reached by an average of 27.5 source probes. This suggests that the computation time for multi-pass transcriptome prediction will be 27.5 times longer than single pass transcriptome prediction if maximum number of source gene per target gene is used.

Ten of the 30 transcriptomes used in the evaluation of single pass transcriptome predictor were used to evaluate the multi-pass transcriptome predictor (see Additional file 1: Table S4 for the microarrays used). Our results suggest that there is no difference in terms of percentage difference (Figure 6A, p-value $=0.076$ ) even though 3 of the 10 predicted transcriptomes (Samples 3, 6, and 7) are

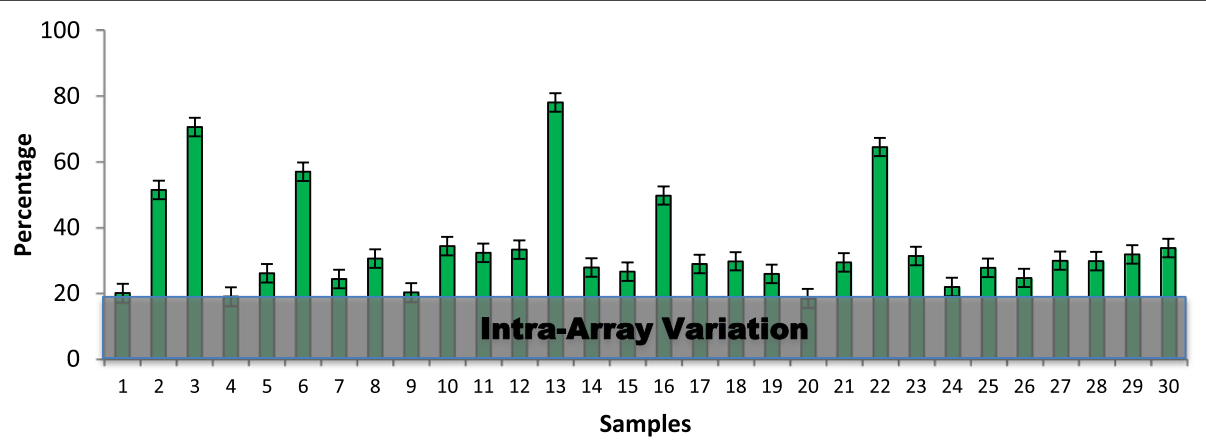

Figure 4 Prediction evaluation test for single-pass transcriptome predictor. Thirty microarrays were used to evaluate the accuracy of the single-pass transcriptome predictor (see Additional file 1: Table S3 for the microarrays used). The number of paths between the source and target genes is 30 to 10000. Error bar denotes standard error. 


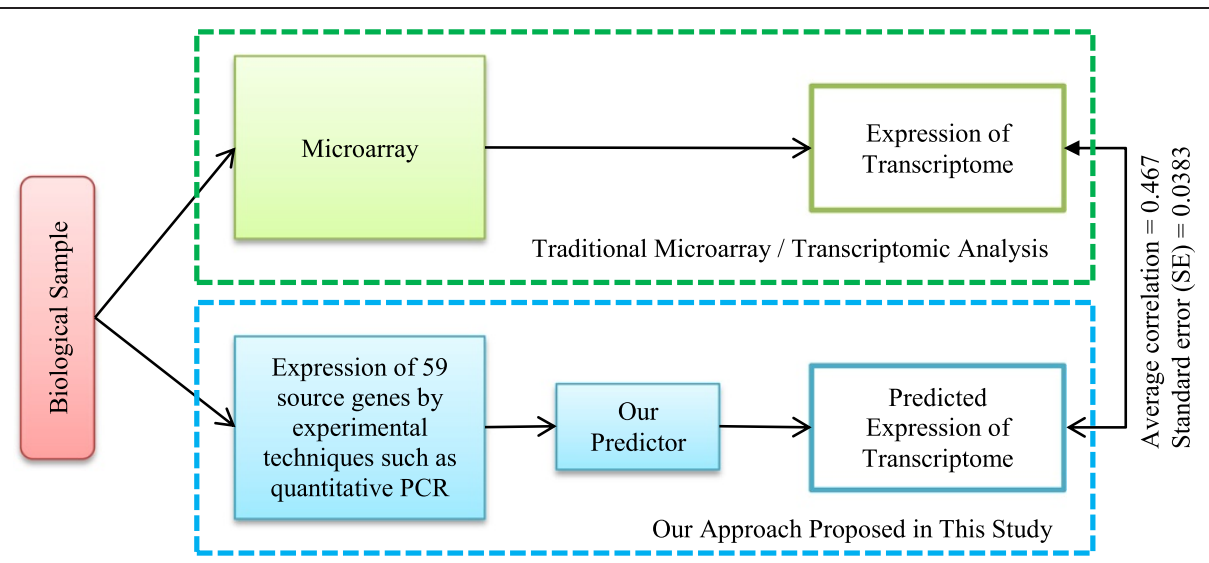

Figure 5 Comparison between our approach (this study) and traditional microarray experiments for analyzing transcriptome. Our approach (this study) demonstrates the ability to predict the expression of the entire transcriptome from the expression of 59 genes; thus, presenting our approach as an economical initial screening of hypotheses before more costly experimental techniques, such as microarrays.

significantly less accurate when predicted by multi-pass method. By examining the standard deviations of the predicted values of each target gene (Figure 6B), multipass method consistently gives higher standard deviation compared to single pass method ( $\mathrm{p}$-value $=3.10 \mathrm{e}-5)$. This suggests that better prediction by multi-pass method in terms of average standard deviations between expected and predicted expression levels of target genes is an artifact as a result of larger standard deviations for the predicted values of each target gene. Correlation between expected and predicted target expression values is significant (average correlation $=0.269, \mathrm{SE}=0.0455$, p-value $=$ $1.13 \mathrm{e}-4)$, which is similar to that previously reported $[8,9]$ but lower than that reported by Fox and Erill [7] and significantly lower from our single pass prediction ( $p$-value $=0.003$, power $=0.999)$. This suggests that multipass transcriptome predictor does not give better prediction compared to single pass transcriptome predictor despite requiring significantly more computational time.

\section{$83 \%$ of perturbation-affected genes predicted within} $40 \%$ error

An important application of transcriptome prediction model is predicting the effects of gene over-expression, knockouts, and environmental stimuli in silico [3]. Overexpressions and knockdowns or under-expressions are collectively known as perturbations. A recent study [67] had modeled the protein concentrations leading to G2 cell cycle checkpoint and validated their simulations of protein level perturbations with published studies.

Our predictor has the potential to estimate the effects of gene perturbation(s). For example, if geneA is overexpressed by 2 times, the affected genes will be the set of genes reachable within 4 jumps of geneA. Our predictor uses a microarray sample as a background transcriptome and performs two predictions. The first prediction predicts the expression values of all reachable genes from the genes of interest before perturbation. Perturbations are carried out by varying the expression values of the genes
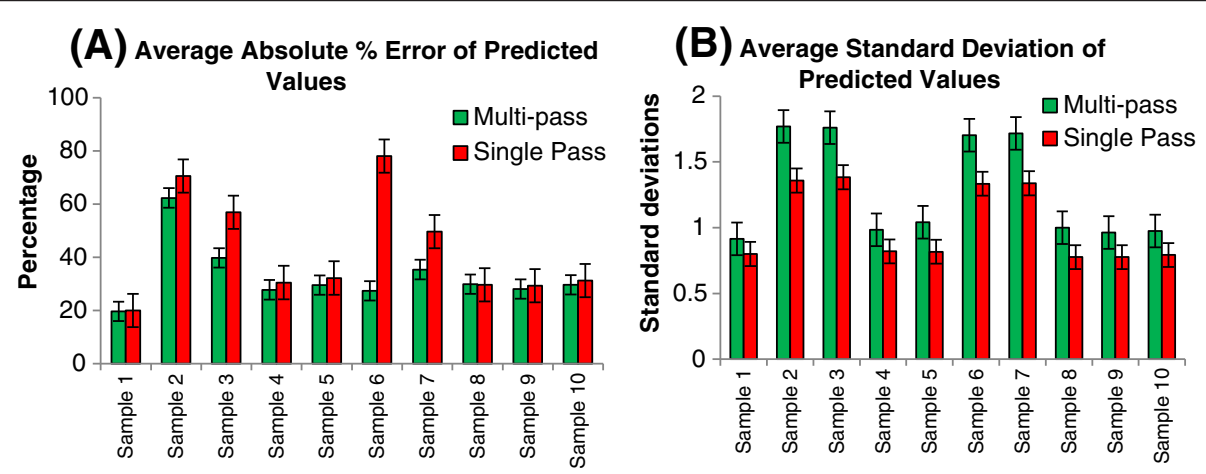

Figure 6 Prediction evaluation test for multi-pass transcriptome predictor. Ten microarrays which had been used for single-pass transcriptome predictor evaluation were used to evaluate multi-pass transcriptome predictor (see Additional file 1: Table S3 for the microarrays used). Error bar denotes standard error. Panel $\mathbf{A}$ shows the average percent difference between the predicted and actual target gene values. Panel $\mathbf{B}$ shows the average standard deviation from each predicted values across different paths. 
of interest before predicting the expression values all reachable genes from the genes of interest after perturbation. Both predictions will provide a predicted value (the mean) and a standard deviation of the affected probes, which allow for standard hypothesis testing and power analysis to be performed.

For evaluation, we identified a background transcriptome, a test transcriptome, and perturbed one or more genes from the background transcriptome to the value of the test transcriptome. Experimentally, if the effects of a 2 times over-expression of geneA in $E$. coli were to be studied, the standard experimental protocol will require an over-expression of geneA using a vector which regulates the expression of geneA under an inducible promoter and compare the transcriptomes of the control sample against the over-expressed sample [68,69]. In our study, the background and test transcriptomes were selected to represent the control and perturbed samples respectively. Three replicates were performed on each of the 6 evaluation tests including single, double and quadruple gene perturbations (see Additional file 1: Table S6 for setup details).

Our results show that at least $73.6 \%$ of the affected genes in single gene over-expression or knockdown are predicted within 40\% error (Figure 7A and B). For double gene over-expression or knockdown, at least $73.8 \%$ of the affected genes are predicted within $40 \%$ of error (Figure $7 \mathrm{C}$ and D). Using single pass prediction, our results show that at least $77.0 \%$ of the genes affected by single gene over-expression with single gene knockdown (2 genes perturbed; Figure 7E) and at least $77.2 \%$ of the genes affected by double gene over-expression with double gene knockdown (4 genes perturbed; Figure 7G) can be predicted within $40 \%$ error. Hence, our results suggest that an average of $83.4 \%(\mathrm{SE}=0.195 \%)$ of perturbationaffected genes can be potentially predicted within $40 \%$ error (which can also be interpreted as within 1.4 folds accuracy).

Comparing single pass versus multi-pass prediction (Figure $7 \mathrm{E}$ versus $7 \mathrm{~F}$, and $7 \mathrm{G}$ versus $7 \mathrm{H}$ ), accuracy between the predicted and actual expression values of the affected genes dropped when multi-pass prediction was used. Statistical comparison between single and multi-pass method shows that this difference is significant ( $\mathrm{p}$-value $=0.0012)$. This is consistent with the findings in our initial multi-pass predictor evaluation. The average correlation between the expression values of affected genes predicted by single-pass method after perturbation is 0.698 with a standard deviation of 0.123 (Additional file 1: Table S7), which is significant ( $\mathrm{p}$-value $=7.44 \mathrm{e}-15$ ). This result is comparable to the correlation of 0.64 (p-value $=0.062)$ reported by McLeay et al. [6], using ChIP-seq, histones and DNase scores to predict gene expression in mammalian cells. This suggests expression values of genes affected by perturbations can be potentially predicted with accuracy comparative to next generation sequencing methods and sequence analyses. This suggests that our predictor may be a useful in silico tool to examine gene perturbations.

Hence, our evaluation also presents itself as a case study of how this predictor can be used. For example, the second replicate of single gene knockdown evaluation corresponds to $56 \%$ knockdown of hydrogenase 2 maturation endopeptidase (hybD), involving in the maturation of hydrogenase 2. Of the 1603 genes affected by this perturbation, 77 genes are directly correlated and 27 genes show more than $3 x$ differences between background expression level and predicted expression level after perturbation. Of the 1526 genes affected between 2 to 4 jumps, 60 are significantly different after Bonferroni correction between predicted expression level before and after perturbation. These 87 genes were analyzed for Gene Ontology enrichment using GOEAST [70]. All 5 significant molecular functions enriched were of carbon/ sugar transferase-typed activity (GOIDs 0008194, 0008378, 0035250, 0016757, and 0016758). This agrees with recent findings associating hydrogenase 2 to hydrogen production during glucose [71] or glycerol fermentation [72].

\section{Expression buffer of hydrogenase 2 maturation endopeptidase (hybD)}

Knowing that $56 \%$ knockdown of $h y b D$ has an impact on the native transcriptome, it is plausible to consider the question of expression buffer. That is, how much expressional variation of $h y b D$ must occur before the underlying native transcriptome is affected? In this case study, we explore this question on a background of E. coli MG1655 pure culture (GSM663167).

Using $10 \%$ stepwise perturbation of $h y b D$ from knockout to $2 \mathrm{x}$ over-expression (Figure $8 \mathrm{~A}$ ), our results suggest that the number of affected genes is symmetrical and fits a quadratic model (paired t-test $\mathrm{p}$-value $=0.182, \mathrm{r}^{2}=0.986$ ) . By solving the roots of the quadratic model, we estimate that an expression between $73.88 \%$ and $124.52 \%$ of the original expression (microarray intensity $=8.535$ ) does not affect the transcriptome of $E$. coli MG1655. In addition, our results also suggest that a reduced model of 5 data points (Figure $8 \mathrm{~B}$ ) is a good estimate (paired t-test $\mathrm{p}$-value $=0.385$ ), suggesting the possibility to reduce computational time if a large number of perturbation analyses are needed. The reduced model estimated an expression buffer between $71.21 \%$ and $128.15 \%$ of 8.535 .

Hence, the predictor may be used to provide estimation to a research question proposed by Selinger et al. [3] - what are the effects of 50\% versus $60 \%$ knockdown of $h y b D$ ? Our results suggest that 148 genes are affected when $h y b D$ is knocked down by $50 \%$ (50\% of original expression) compared to 307 genes when $h y b D$ is knocked down by $60 \%$ ( $40 \%$ of original expression). 


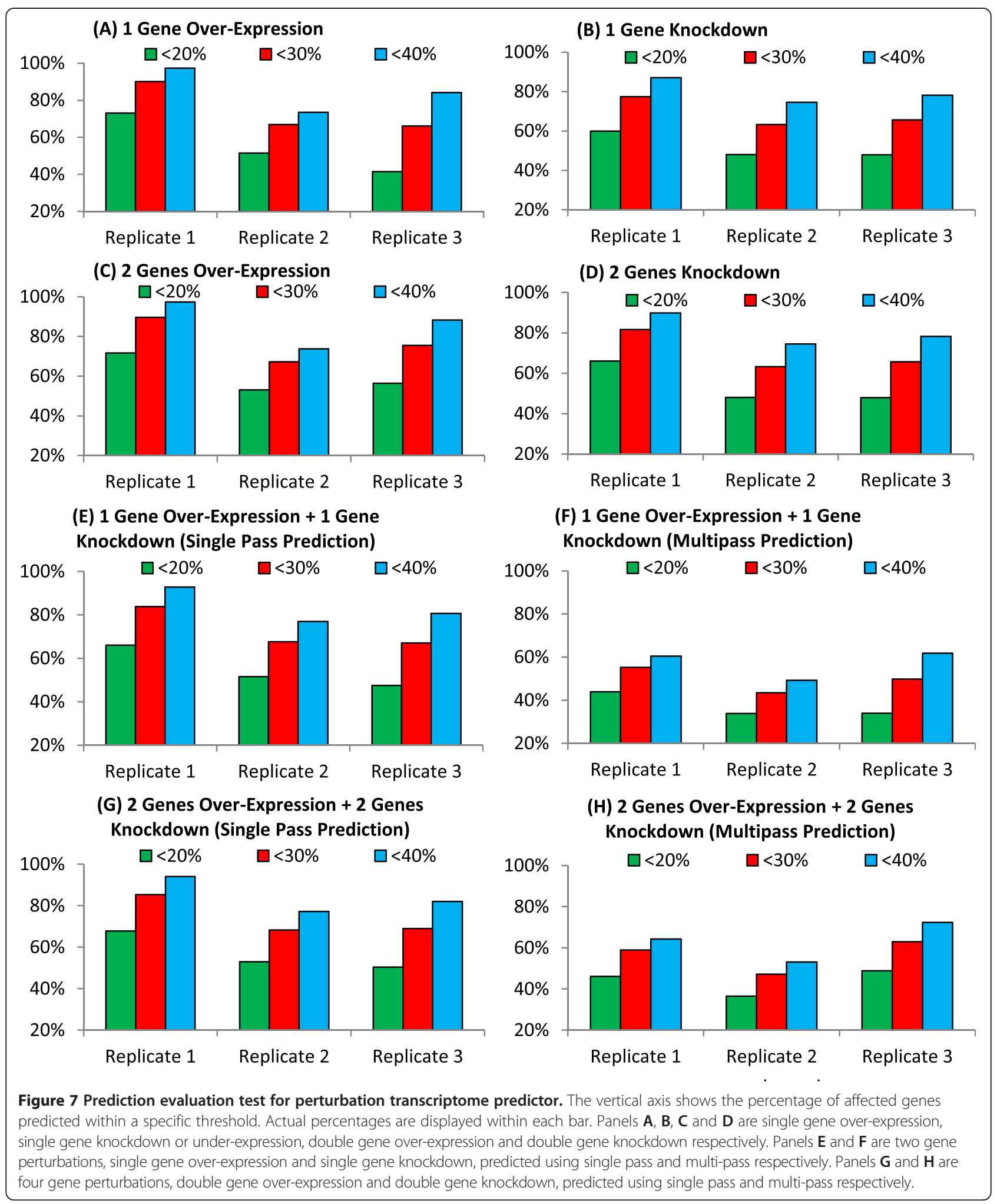

This study provides baseline and test cases for future work In this study, we present a potential means to predict virtually the entire transcriptome from a set of 59 source genes, which may be useful for synthetic biologists to predict the effects of transgene [33]. In addition, our predictor has the potential to examine the effect of one or more genes when their expression is/are changed [3] and shown to perform comparatively to previous studies 
$\mathbf{A}$

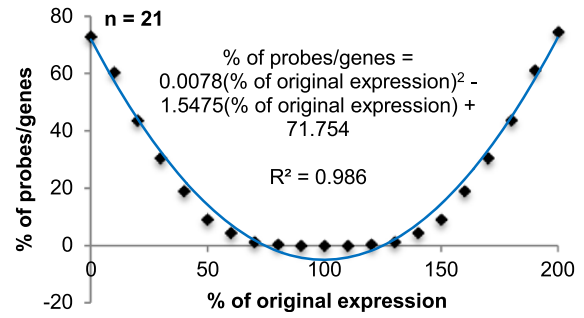

B

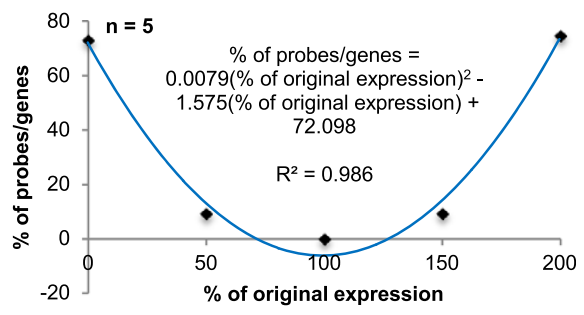

Figure 8 Percentage of reachable genes affected by varying levels of hydrogenase 2 maturation endopeptidase (hybD) expressions. Panel A shows 10\% stepwise expression variation from total knockout to 2x over-expression (21 data points). Panel B shows 50\% stepwise expression variation from total knockout to $2 x$ over-expression ( 5 data points). Both models are not statistically different from each other (paired t-test $p$-value $=0.385$ ).

on predicting prokaryotic gene expressions using sequence features such as codon usage bias [7,9].

Using the simplest statistical model to relate the expression values of 2 genes, this study acts as a baseline for future work. Non-linear or higher-order regression models [18-21], may be used to improve prediction accuracy. The prediction accuracy may also be improved with additional microarray data as they come online or including data from less noisy sources, such as from RNA sequencing. At the same time, we had described the test cases used (see Additional file 1) throughout this study, which can be used to evaluate future improvements to this work.

\section{Conclusion}

In this study, we demonstrate that the transcriptome of E. coli can be potentially predicted from a set of marker gene expressions or from known perturbation. The former enables thousands of gene expressions to be predicted from a small set of known gene expressions while the latter enables in silico evaluation of the effects gene perturbation(s) such as gene over-expression(s) and/or under-expression(s). Hence, we present a potential means to predict an entire transcriptome and a tool to estimate the effects of gene perturbations for $E$. coli, which will aid biologists in hypothesis development. This study forms the baseline for future work in using gene co-expression network for gene expression prediction.

\section{Methods}

\section{Construction of co-expression network and regression models}

The CEL files of $605 \mathrm{E}$. coli microarrays across 40 experiments were downloaded from NCBI Gene Expression Omnibus (see Additional file 1: Table S1 for a list of series used) and RMA normalized using Affymetrix Expression Console. Pairwise permutations of Pearson's correlation were calculated and the expression values for the pair of genes were fitted into first order linear regression equation in the form of $\operatorname{Gene}(x)=b_{1} \operatorname{Gene}(y)+b_{0}$. Pairs with absolute Pearson's correlation of more than 0.75 were retained for building co-expression network using Network $X$ where the nodes were the genes and an edge existed between the nodes when the absolute Pearson's correlation between the two genes was more than 0.75 .

\section{Predicting transcriptome}

Two transcriptome predictors, single pass and multipass, were implemented. The difference between the two predictors is that the single pass predictor performed one prediction per target gene whereas the multi-pass predictor allowed a target gene to be predicted using 2 or more source genes. Thus, in single pass prediction, a target gene expression will be estimated from one or more paths from the source gene expression. Once a target gene expression is estimated, its expression will not be re-estimated even though the target gene can be predicted by more than one source gene. The sequence of target gene expression prediction is dependent on the sequence of source gene expression and the number of jumps (degree) from the source gene. For example, if a target gene can be estimated by 2 different source genes at 3 degrees, the first source gene will be used to estimate the target gene expression in single pass predictor. If a target gene can be estimated by 2 different source genes at 3 and 4 degrees respectively, the target gene expression will be estimated by the source gene at 3 degrees instead of the source gene at 4 degrees in single pass predictor. In multi-pass predictor, both source genes will be used to estimate the target gene expression regardless of positional sequence of the source gene list or the degrees between source genes and the target gene. Given a list of source genes (marker genes) and their expression values, the transcriptome predictors predict all genes reachable within 4 jumps using a loop over the linear regression models. For example, if Gene(A) is a source gene with known expression and is connected to Gene (C) via Gene(B), then the expression of Gene(B) can be predicted by the known expression of Gene(A) by linear regression between $\operatorname{Gene}(\mathrm{A})$ and $\operatorname{Gene}(\mathrm{B})$. Bringing this a 
step forward, the expression of $\mathrm{Gene}(\mathrm{C})$ can be predicted by the predicted expression of Gene(B) by linear regression between $\operatorname{Gene}(\mathrm{B})$ and $\operatorname{Gene}(\mathrm{C})$. Therefore, the expression level of $\operatorname{Gene}(\mathrm{C})$ can be predicted as $\operatorname{Gene}(\mathrm{C})=\mathrm{b}_{1, \mathrm{~B}-\mathrm{C}}\left(\mathrm{b}_{1, \mathrm{~A}-}\right.$ $\left.{ }_{B} G e n e(A)+b_{0, A-B}\right)+b_{0, B-C}$ where $b_{1, A-B}$ and $b_{0, A-B}$ is the first-order linear regression gradient and intercept between Gene(A) and Gene(B) respectively, and $\mathrm{b}_{1, \mathrm{~B}-\mathrm{C}}$ and $\mathrm{b}_{0, \mathrm{~B}-\mathrm{C}}$ is the first-order linear regression gradient and intercept between Gene(B) and $\operatorname{Gene}(\mathrm{C})$ respectively. As there could be more than one path between any source and target genes via different intermediary genes, there could be more than one predicted expression values. The predictor would report the arithmetic mean and standard deviation of the predicted values (see Figure 1).

\section{Predicting the effects of perturbation(s)}

A list of perturbations was given as ratio of the original expression values, for example, 1.8 times of Gene(A) and 0.4 times of Gene(B). The predictor estimated the effects of perturbations by a two-pass transcriptome prediction where the first pass predicted the expression values of all affected target genes within 4 jumps using the original expression values of the background transcriptome $[1 \times$ Gene (A) and $1 \times$ Gene(B)], followed by a second pass using the perturbed values from the background transcriptome [1.8× Gene(A) and $0.4 \times$ Gene(B)]. As a result, each perturbation runs using different combinations of perturbed genes might have different numbers of affected target genes.

\section{Evaluating predictors}

The single pass and multi-pass transcriptome predictors were evaluated using 30 and 10 microarrays that were not used for model building respectively (see Additional file 1: Table S3 for arrays used and labeling). Perturbation prediction was evaluated using six types of perturbations (1. Single gene over-expression. 2. Single gene knockdown. 3. Double gene over-expression. 4. Double gene knockdown. 5. Single gene over-expression with single gene knockdown. 6. Double gene over-expression with double gene knockdown.) on 3 replicates (see Additional file 1: Table S4 for detailed setup and microarrays used). For each microarray, the expression values of 59 genes were extracted and used as source genes to predict all reachable genes, known as target genes, within 4 jumps. The target genes consisted of adjacent genes (one jump from source genes) and non-adjacent genes (two to four jumps from source genes). As there would be only one path from source gene to adjacent gene, standard deviation would not be calculated and only non-adjacent genes would be used to evaluate the predictors. The accuracy of prediction was determined by the number of standard deviations and the percentage difference between the expected expression value (from the microarray data) and the average predicted values.

\section{Additional file}

Additional file 1: Table S1. Microarray data series for model building Table S2. Internal consistency of microarray values. Table S3. List of 59 source probes. Table S4. Microarrays used to evaluate the accuracy of single pass and multi-pass transcriptome predictors. Table S5. Comparison between quantitative PCR findings of Kendall et al. [45] and gene expression prediction. Table S6. Perturbation predictor evaluation setup. Table S7. Correlations between predicted and expected expression values of genes affected by perturbation(s). Figure S1. Distribution of path length.

Competing interests

The authors declare that they have no competing interests.

\section{Authors' contributions}

$\mathrm{ML}$ and CLP conceived of the project. ML performed the research and analyzed the data. CLP provided essential comments and guidance. The manuscript was written by ML and edited by CLP. All authors read and approved the final manuscript.

\section{Acknowledgement}

This work is supported by Ministry of Education (MOE) Singapore Tier 2 grant.

Received: 28 November 2013 Accepted: 9 May 2014

Published: 13 May 2014

\section{References}

1. Medema MH, van Raaphorst R, Takano E, Breitling R: Computational tools for the synthetic design of biochemical pathways. Nat Rev Microbiol 2012, 10(3):191-202.

2. Schellenberger J, Que R, Fleming RM, Thiele I, Orth JD, Feist AM, Zielinski DC, Bordbar A, Lewis NE, Rahmanian S, Kang J, Hyduke DR, Palsson BØ: Quantitative prediction of cellular metabolism with constraint-based models: the COBRA Toolbox v2.0. Nat Protoc 2011, 6(9):1290-1307.

3. Selinger DW, Wright MA, Church GM: On the complete determination of biological systems. Trends Biotechnol 2003, 21(6):251-254.

4. Chikina MD, Huttenhower C, Murphy CT, Troyanskaya OG: Global prediction of tissue-specific gene expression and context-dependent gene networks in Caenorhabditis elegans. PLoS Comput Biol 2009, 5:e1000417.

5. Ouyang Z, Zhou Q, Wong WH: ChIP-Seq of transcription factors predicts absolute and differential gene expression in embryonic stem cells. Proc Natl Acad Sci U S A 2009, 106(51):21521-21526.

6. McLeay RC, Lesluyes T, Cuellar Partida G, Bailey TL: Genome-wide in silico prediction of gene expression. Bioinformatics 2012, 28(21):2789-2796.

7. Fox JM, Erill I: Relative codon adaptation: a generic codon bias index for prediction of gene expression. DNA Res 2010, 17:185-196.

8. Roymondal U, Das S, Sahoo S: Predicting gene expression level from relative codon usage bias: an application to Escherichia coli genome. DNA Res 2009, 16(1):13-30.

9. Das S, Roymondal U, Chottopadhyay B, Sahoo S: Gene expression profile of the cynobacterium synechocystis genome. Gene 2012, 497:344-352.

10. Menashe I, Grange P, Larsen EC, Banerjee-Basu S, Mitra PP: Co-expression profiling of autism genes in the mouse brain. PLoS Comput Biol 2013, 9(7):e1003128.

11. Liu R, Liao J, Yang M, Sheng J, Yang H, Wang Y, Pan E, Guo W, Pu Y, Kim SJ, Yin L: The cluster of miR-143 and miR-145 affects the risk for esophageal squamous cell carcinoma through co-regulating fascin homolog 1. PLOS One 2012, 7(3):e33987.

12. Moreno-Sanchez N, Rueda J, Carabano MJ, Reverter A, McWilliam S, Gonzalez C, Diaz C: Skeletal muscle specific genes networks in cattle. Funct Integr Genomics 2010, 10(4):609-618.

13. Liao BY, Zhang J: Evolutionary conservation of expression profiles between human and mouse orthologous genes. Mol Biol Evol 2006, 23(3):530-540.

14. Ling MH, Ban Y, Wen H, Wang SM, Ge SX: Conserved expression of natural antisense transcripts in mammals. BMC Genomics 2013, 14(1):243.

15. Torkamani A, Dean B, Schork NJ, Thomas EA: Coexpression network analysis of neural tissue reveals perturbations in developmental processes in schizophrenia. Genome Res 2010, 20(4):403-412.

16. Childs KL, Davidson RM, Buell CR: Gene coexpression network analysis as a source of functional annotation for rice genes. PLoS One 2011, 6:e22196. 
17. Ray M, Zhang W: Analysis of Alzheimer's disease severity across brain regions by topological analysis of gene co-expression networks. BMC Syst Biol 2010, 4:136

18. Kadarmideen HN, Watson-Haigh NS: Building gene co-expression networks using transcriptomics data for systems biology investigations: Comparison of methods using microarray data. Bioinformation 2012, 8(18):855-861.

19. Obayashi T, Kinoshita K: Rank of correlation coefficient as a comparable measure for biological significance of gene coexpression. DNA Res 2009 16(5):249-260.

20. Zhao W, Langfelder P, Fuller T, Dong J, Li A, Hovarth S: Weighted gene coexpression network analysis: state of the art. J Biopharm Stat 2010, 20(2):281-300.

21. Furlotte NA, Kang HM, Ye C, Eskin E: Mixed-model coexpression: calculating gene coexpression while accounting for expression heterogeneity. Bioinformatics 2011, 27:i288-i294

22. Jupiter $\mathrm{D}$, Chen $\mathrm{H}$, VanBuren V: STARNET 2: a web-based tool for accelerating discovery of gene regulatory networks using microarray co-expression data. BMC Bioinformatics 2009, 10:332.

23. Wada M, Takahashi H, Altaf-Ul-Amin M, Nakamura K, Hirai MY, Ohta D, Kanaya S: Prediction of operon-like gene clusters in the Arabidopsis thaliana genome based on co-expression analysis of neighboring genes. Gene 2012, 503(1):56-64.

24. Chay ZE, Lee $\mathrm{CH}$, Lee KC, Oon JS, Ling MH: Russel and Rao coefficient is a suitable substitute for Dice coefficient in studying restriction mapped genetic distances of Escherichia coli. Comput Math Biol 2010, 1:1.

25. Park SJ, Nakai K: A regression analysis of gene expression in ES cells reveals two gene classes that are significantly different in epigenetic patterns. BMC Bioinformatics 2011, 12(Suppl 1):S50

26. Oeder $S$, Mages J, Flicek $P$, Lang R: Uncovering information on expression of natural antisense transcripts in Affymetrix MOE430 datasets. BMC Genomics 2007, 8:200.

27. Reverter A, Barris W, Moreno-Sanchez N, McWilliam S, Wang YH, Harper GS, Lehnert SA, Dalrymple BP: Construction of gene interaction and regulatory networks in bovine skeletal muscle from expression data. Aust J Exp Agric 2005, 45:821-829.

28. Shi W, Banerjee A, Ritchie ME, Gerondakis S, Smyth GK: Illumina WG-6 BeadChip strips should be normalized separately. BMC Bioinformatics 2009, 10:372.

29. Chain B, Bowen H, Hammond J, Posch W, Rasaiyaah J, Tsang J, Noursadeghi M: Error, reproducibility and sensitivity: a pipeline for data processing of Agilent oligonucleotide expression arrays. BMC Bioinformatics 2010, 11:344.

30. Anderson T, Wulfkuhle J, Liotta L, Winslow RL, Petricoin E III: Improved reproducibility of reverse-phase protein microarrays using array microenvironment normalization. Proteomics 2009, 9(24):5562-5566.

31. Gyorffy B, Molnar B, Lage H, Szallasi Z, Eklund AC: Evaluation of microarray preprocessing algorithms based on concordance with RT-PCR in clinical samples. PLoS One 2009, 4:e5645.

32. Wu D, Hu Y, Tong S, Williams BR, Smyth GK, Gantier MP: The use of miRNA microarrays for the analysis of cancer samples with global miRNA decrease. RNA 2013, 19(7):876-888.

33. Porcar M, Danchin A, de Lorenzo V, Dos Santos VA, Krasnogor N, Rasmussen S, Moya A: The ten grand challenges of synthetic life. Syst Synthetic Biol 2011, 5(1-2):1-9.

34. Alteri CJ, Lindner JR, Reiss DJ, Smith SN, Mobley HL: The broadly conserved regulator PhoP links pathogen virulence and membrane potential in Escherichia coli. Mol Microbiol 2011, 82(1):145-163.

35. Bansal T, Jesudhasan P, Pillai S, Wood TK, Jayaraman A: Temporal regulation of enterohemorrhagic Escherichia coli virulence mediated by autoinducer-2. Appl Microbiol Biotechnol 2008, 78(5):811-819.

36. Bansal T, Kim DN, Slininger T, Wood TK, Jayaraman A: Human intestinal epithelial cell-derived molecule(s) increase enterohemorrhagic Escherichia coli virulence. FEMS Immunol Med Microbiol 2012, 66(3):399-410.

37. Chattopadhyay MK, Chen W, Tabor H: Escherichia coli glutathionylspermidine synthetase/amidase: phylogeny and effect on regulation of gene expression. FEMS Microbiol Lett 2013, 338(2):132-140.

38. Chen T, Wang J, Zeng L, Li R, Li J, Chen Y, Lin Z: Significant rewiring of the transcriptome and proteome of an Escherichia coli strain harboring a tailored exogenous global regulator IrrE. PLoS One 2012, 7(7):e37126.

39. Cho BK, Federowicz SA, Embree M, Park YS, Kim D, Palsson BO: The PurR regulon in Escherichia coli K-12 MG1655. Nucleic Acids Res 2011, 39(15):6456-6464
40. Chu W, Zere TR, Weber MM, Wood TK, Whiteley M, Hidalgo-Romano B, Valenzuela E Jr, McLean RJ: Indole production promotes Escherichia coli mixed-culture growth with Pseudomonas aeruginosa by inhibiting quorum signaling. Appl Environ Microbiol 2012, 78(2):411-419.

41. Durand S, Storz G: Reprogramming of anaerobic metabolism by the FnrS small RNA. Mol Microbiol 2010, 75(5):1215-1231.

42. Habdas BJ, Smart J, Kaper JB, Sperandio V: The LysR-type transcriptional regulator QseD alters type three secretion in enterohemorrhagic Escherichia coli and motility in K-12 Escherichia coli. J Bacteriol 2010, 192(14):3699-3712.

43. Hensley MP, Gunasekera TS, Easton JA, Sigdel TK, Sugarbaker SA, Klingbeil L, Breece RM, Tierney DL, Crowder MW: Characterization of Zn(II)-responsive ribosomal proteins YkgM and L31 in E. coli. J Inorg Biochem 2012, 111:164-172.

44. Hidalgo G, Ponton A, Fatisson J, O'May C, Asadishad B, Schinner T, Tufenkji $\mathrm{N}$ : Induction of a state of iron limitation in uropathogenic Escherichia coli CFT073 by cranberry-derived proanthocyanidins as revealed by microarray analysis. App/ Environ Microbiol 2011, 77(4):1532-1535.

45. Kendall MM, Rasko DA, Sperandio V: Global effects of the cell-to-cell signaling molecules autoinducer-2, autoinducer-3, and epinephrine in a luxS mutant of enterohemorrhagic Escherichia coli. Infect Immun 2007 75(10):4875-4884

46. Kim Y, Wang X, Zhang XS, Grigoriu S, Page R, Peti W, Wood TK: Escherichia coli toxin/antitoxin pair MqsR/MqsA regulate toxin CspD. Environ Microbiol 2010, 12(5):1105-1121.

47. Lee J, Zhang XS, Hegde M, Bentley WE, Jayaraman A, Wood TK: Indole cell signaling occurs primarily at low temperatures in Escherichia coli. ISME J 2008, 2(10):1007-1023.

48. Lee J, Hiibel SR, Reardon KF, Wood TK: Identification of stress-related proteins in Escherichia coli using the pollutant cis-dichloroethylene J Appl Microbiol 2010, 108(6):2088-2102.

49. Li Y, Zhang Y: PhoU is a persistence switch involved in persister formation and tolerance to multiple antibiotics and stresses in Escherichia coli. Antimicrob Agents Chemother 2007, 51(6):2092-2099.

50. Ma Q, Wood TK: OmpA influences Escherichia coli biofilm formation by repressing cellulose production through the CpxRA two-component system Environ Microbiol 2009, 11(10):2735-2746.

51. Moon K, Gottesman S: A PhoQ/P-regulated small RNA regulates sensitivity of Escherichia coli to antimicrobial peptides. Mol Microbiol 2009, 74(6):1314-1330.

52. Nakanishi Y, Fukuda S, Chikayama E, Kimura Y, Ohno H, Kikuchi J: Dynamic omics approach identifies nutrition-mediated microbial interactions. J Proteome Res 2011, 10(2):824-836.

53. Nobre LS, Al-Shahrour F, Dopazo J, Saraiva LM: Exploring the antimicrobial action of a carbon monoxide-releasing compound through wholegenome transcription profiling of Escherichia coli. Microbiology 2009 155(Pt 3):813-824.

54. Reading NC, Rasko D, Torres AG, Sperandio V: A transcriptome study of the QseEF two-component system and the QseG membrane protein in enterohaemorrhagic Escherichia coli O157: H7. Microbiology 2010, 156(Pt 4):1167-1175.

55. Strader MB, Costantino N, Elkins CA, Chen CY, Patel I, Makusky AJ, Choy JS, Court DL, Markey SP, Kowalak JA: A proteomic and transcriptomic approach reveals new insight into beta-methylthiolation of Escherichia coli ribosomal protein S12. Mol Cell Proteomics 2011, 10(3):M110 005199.

56. Traxler MF, Zacharia VM, Marquardt S, Summers SM, Nguyen HT, Stark SE, Conway T: Discretely calibrated regulatory loops controlled by ppGpp partition gene induction across the 'feast to famine' gradient in Escherichia coli. Mol Microbiol 2011, 79(4):830-845

57. Waters LS, Sandoval M, Storz G: The Escherichia coli MntR miniregulon includes genes encoding a small protein and an efflux pump required for manganese homeostasis. J Bacteriol 2011, 193(21):5887-5897.

58. Yang C, Huang TW, Wen SY, Chang CY, Tsai SF, Wu WF, Chang CH: Genome-wide PhoB binding and gene expression profiles reveal the hierarchical gene regulatory network of phosphate starvation in Escherichia coli. PLoS One 2012, 7(10):e47314.

59. Abadia E, Zhang J, Ritacco V, Kremer K, Ruimy R, Rigouts L, Gomes HM, Elias AR, Fauville-Dufaux M, Stoffels K, Rasolofo-Razanamparany V, de Garcia Viedma D, Herranz M, Al-Hajoj S, Rastogi N, Garzelli C, Tortoli E, Suffys PN, Van Soolingen D, Refrégier G, Sola C: The use of microbead-based spoligotyping for Mycobacterium tuberculosis complex to evaluate the quality of the conventional 
method: providing guidelines for Quality Assurance when working on membranes. BMC Infect Dis 2011, 11:110.

60. Hermida L, Poussin C, Stadler MB, Gubian S, Sewer A, Gaidatzis D, Hotz HR, Martin F, Belcastro V, Cano S, Peitsch MC, Hoeng J: Confero: an integrated contrast data and gene set platform for computational analysis and biological interpretation of omics data. BMC Genomics 2013, 14:514.

61. Tomlinson C, Thimma M, Alexandrakis S, Castillo T, Dennis JL, Brooks A, Bradley T, Turnbull C, Blaveri E, Barton G, Chiba N, Maratou K, Soutter P, Aitman T, Game L: MiMiR-an integrated platform for microarray data sharing, mining and analysis. BMC Bioinformatics 2008, 9:379.

62. Tseng GC, Ghosh D, Feingold E: Comprehensive literature review and statistical considerations for microarray meta-analysis. Nucleic Acids Res 2012, 40(9):3785-3799.

63. Chia CY, Lim CW, Leong WT, Ling MH: High expression stability of microtubule affinity regulating kinase 3 (MARK3) makes it a reliable reference gene. IUBMB Life 2010, 62(3):200-203.

64. Heng SS, Chan OY, Keng BM, Ling MH: Glucan Biosynthesis Protein G Is a Suitable Reference Gene in Escherichia coli K-12. ISRN Microbiol 2011, 2011:469053.

65. Hira ZM, Trigeorgis G, Gillies DF: An algorithm for finding biologically significant features in microarray data based on a priori manifold learning. PLoS One 2014, 9(3):e90562.

66. Wilczynski B, Liu YH, Yeo ZX, Furlong EE: Predicting Spatial and Temporal Gene Expression Using an Integrative Model of Transcription Factor Occupancy and Chromatin State. PLoS Comput Biol 2012, 8(12):e1002798.

67. Kesseler KJ, Blinov ML, Elston TC, Kaufmann WK, Simpson DA: A predictive mathematical model of the DNA damage G2 checkpoint. J Theor Biol 2013, 320:159-169.

68. Escudero JM, Haller JL, Clay CM, Escudero KW: Microarray analysis of Foxl2 mediated gene regulation in the mouse ovary derived KK1 granulosa cell line: Over-expression of Foxl2 leads to activation of the gonadotropin releasing hormone receptor gene promoter. J Ovarian Res 2010, 3:4

69. Jiang SY, Bhalla R, Ramamoorthy R, Luan HF, Venkatesh PN, Cai M, Ramachandran S: Over-expression of OSRIP18 increases drought and salt tolerance in transgenic rice plants. Transgenic Res 2012, 21:785-795.

70. Zheng Q, Wang XJ: GOEAST: a web-based software toolkit for Gene Ontology enrichment analysis. Nucleic Acids Res 2008, 36(Web Server issue):W358-W363.

71. Maeda T, Sanchez-Torres V, Wood TK: Enhanced hydrogen production from glucose by metabolically engineered Escherichia coli. Appl Microbiol Biotechnol 2007, 77(4):879-890.

72. Trchounian K, Soboh B, Sawers RG, Trchounian A: Contribution of Hydrogenase 2 to stationary phase $\mathrm{H}(2)$ production by Escherichia coli during fermentation of glycerol. Cell Biochem Biophys 2012, 66(1):103-108

doi:10.1186/1471-2105-15-140

Cite this article as: Ling and Poh: A predictor for predicting Escherichia coli transcriptome and the effects of gene perturbations. BMC Bioinformatics 2014 15:140.

\section{Submit your next manuscript to BioMed Central and take full advantage of:}

- Convenient online submission

- Thorough peer review

- No space constraints or color figure charges

- Immediate publication on acceptance

- Inclusion in PubMed, CAS, Scopus and Google Scholar

- Research which is freely available for redistribution 